\title{
Rash Maculo-Papular, CTCAE
}

National Cancer Institute

\section{Source}

National Cancer Institute. Rash Maculo-Papular, CT CAE. NCI Thesaurus. Code C143799.

A disorder characterized by the presence of macules (flat) and papules (elevated). Also known as morbillform rash, it is one of the most common cutaneous adverse events, frequently affecting the upper trunk, spreading centripetally and associated with pruritis. 